\section{Visión Electrónica \\ Más que un estado sólido}

https://revistas.udistrital.edu.co/index.php/visele

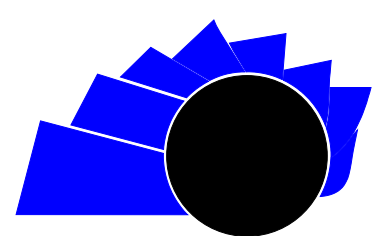

VISIÓN ELECTRÓNICA

A VISIÓN DE CASO

\title{
Prototipo para monitorización de signos vitales en espacios confinados
}

\author{
Prototype for Monitoring Vital Signs in Confined Spaces
}

\section{Tatiana Salamanca. ${ }^{1}$}

\section{INFORMACIÓN DEL ARTÍCULO}

\section{Historia del artículo:}

Enviado: 24/09/2017

Recibido: 21/11/2017

Aceptado: 03/01/2018

Palabras clave:

Comunicación serial

Espacios confinados

Monitoreo

Signos vitales

\section{Open access

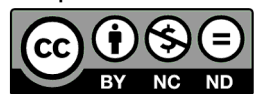

Keywords:

Serial communication

Confined spaces

Monitoring

Vital signs

\section{RESUMEN}

Debido a que los espacios que poseen ventilación desfavorable y accesos reducidos ponen en riesgo la vida de los operarios que los ocupan, identificar la trazabilidad y estabilidad del trabajador en el interior de estos es un asunto pertinente en Ingeniería en Higiene y Salud Ocupacional; por lo anterior, el presente artículo describe la investigación que condujo al diseño e implementación de un prototipo para adquisición, transmisión y monitorización de signos vitales en trabajadores que laboran en espacios confinados. Las variables sujetas a mediciones periódicas son temperatura, pulso cardiaco y aceleración; los datos adquiridos, filtrados digitalmente, se transmiten por el dispositivo a un servidor web, donde se visualizan a través de un vínculo de direccionamiento a internet usando una red Wifi. La monitorización eficaz resultante permite evaluar el estado del trabajador, por lo que se puede prever y prevenir diversidad de anomalías que se presentan en su entorno laboral.

\section{ABSTRACT}

Because the open spaces of ventilation and reduced accesses endanger the life of the workers who occupy them, identify the traceability and the stability of the worker inside it is a relevant topic in the Engineering in Hygiene and Occupational Health. Therefore, this article describes the research that led to the design and implementation of a prototype for the acquisition, transmission and monitoring of vital signs in workers who work in confined spaces. The variables subject to periodic measurements are: temperature, heart rate and acceleration; the acquired data, filtered digitally, is transmitted by the device to a web server, and it is visualized through an Internet address link using a Wi-Fi network. The efficient monitoring of the dispositive allows evaluating the worker's condition, so that can it foresee and prevent the diversity of anomalies that appear in his work environment.

\footnotetext{
${ }^{1}$ Bioingeniera, Universidad El Bosque. Correo electrónico: tsalamanca@unbosque.edu.co, tpsalamanca@outlook.com.
} 


\section{Introducción}

Actualmente, tanto en el ámbito comercial como en el académico, existe una diversidad de artefactos que monitorizan variables humanas tales como el Apple Watch, la manilla Fitbit, entre otros; sin embargo, se ha evidenciado que son de uso recreativo o deportivo [1-4]. No obstante, la empresa que más cercana ha estado de realizar un prototipo orientado a la seguridad industrial ha sido Mapfre, pero su propuesta dista de lo que se formula en la presente investigación - desde el punto de vista de la funcionalidad para espacios con características específicas - Es por lo anterior que, teniendo en cuenta la importancia de la elaboración de un prototipo de seguridad industrial, hay que considerar la existencia de diversidad de labores estimadas como trabajos de alto riesgo, entre las que se encuentran los trabajos en espacios confinados, caracterizados por poseer aberturas limitadas de entrada y salida y una ventilación natural desfavorable [5].

Ahora bien, teniendo en cuenta las características de un espacio confinado, el presente artículo se enfoca especialmente en la elaboración de un dispositivo de seguridad industrial aplicado en tales espacios. Este artefacto tiene como finalidad prevenir accidentes laborales por los diversos cambios de temperaturas, acumulación de gases o caídas en los operarios que deben ingresar en estos espacios confinados, a partir de una monitorización constante. Por lo anterior, se realizó un prototipo inicial que permite la monitorización de la temperatura del espacio confinado, la posición en un plano coordenado que permite establecer si la persona se encuentra de pie o ha sufrido un desmayo y, además, monitorea en tiempo real los signos vitales, la temperatura corporal y pulso cardiaco en los trabajadores que ingresan en ellos [6-10]. Cada una de las variables mencionadas será identificada o podrá observarse su comportamiento a través de un servidor web, el cual puede ser monitoreado por el personal capacitado de la empresa.

La estructura del documento es la siguiente: en primer lugar se caracterizan los espacios confinados desde el punto de vista de los trabajadores para establecer los materiales y métodos utilizados en las cuatro fases de desarrollo - selección de sensores, diseño y desarrollo del prototipo experimental, filtrado digital y eliminación de ruido y los ensayos de laboratorio-; posteriormente se exhibe el prototipo resultante y, finalmente, se establecen las conclusiones de la investigación.

\section{Materiales y métodos}

Los trabajadores en espacios confinados pueden verse severamente afectados por las condiciones a las cuales se encuentran sometidos debido a la ventilación desfavorable. Uno de los factores más comunes de riesgo es la inhalación de gases tóxicos, pues se evaporan sustancias como sulfatos, óxidos, nitratos, entre otros, los cuales pueden producir deficiencia de oxígeno al operario que se encuentre en el lugar. En tales condiciones, la sangre no podrá enriquecerse suficientemente de oxigeno $\left(\mathrm{O}_{2}\right)$ como para satisfacer las necesidades básicas del organismo, conllevando al desencadenamiento de diversos daños fisiológicos que se dan dependiendo de la cantidad de oxígeno contenido en las células. Las consecuencias sobre el músculo cardiaco tienden a que disminuya su frecuencia de bombeo y, a raíz de ello, cada uno de los órganos y tejidos del cuerpo empiezan a fallar, en cuestión de segundos se generan daños a nivel metabólico, circulatorio y cerebral, hasta el punto de producirse daños severos que desencadenan, en el peor de los casos, parálisis total del individuo.

Adicionalmente, en los espacios confinados, en especial en las minas y en las torres de refinería de petróleo, hay diversidad de gases licuados como nitrógeno líquido, argón líquido o dióxido de carbono líquido; en el caso de que se evapore un litro de estos líquidos, se genera aproximadamente 850 litros de gas, este volumen enorme de gases puede rápidamente llevar a una deficiencia de oxígeno a menos que haya una ventilación adecuada, sin embargo, las condiciones de ventilación en estos lugares son mínimas y de difícil acceso [11-13].

En suma, la situación es crítica porque los trabajadores en espacios confinados se exponen a diversidad de peligros debido a las condiciones de su entorno, aunado a que no es fácil detectar la presencia de los gases mencionados anteriormente, tampoco podrían recibir asistencia oportuna en caso de un accidente debido a la distancia que existe entre la zona de trabajo y las instalaciones en superficie. Por lo expuesto, las condiciones de los espacios confinados generan una alta tasa de mortalidad en trabajadores, específicamente en las torres de refinería de petróleo en las que mueren 200 personas anualmente, según datos arrojados por el Instituto Nacional para la Seguridad y Salud Ocupacional (NIOSH, por sus siglas en inglés); de estas muertes registradas, dos tercios se deben a atmósferas peligrosas, causantes de asfixia en las personas. Por lo anterior, según la Administración para la Seguridad y Salud Laboral (OSHA, por sus siglas en inglés) la prevención es la mejor herramienta para evitar que se produzcan accidentes en los entornos laborales; en este 
sentido, a lo largo de la presente investigación se propone el diseño de un prototipo de dispositivo que monitoree pulso cardiaco y la temperatura de los trabajadores que se encuentren en torres de destilación de petróleo, acompañado de un acelerómetro y un botón de pánico (Figura 1).

Figura 1: Diagrama de bloques [1].

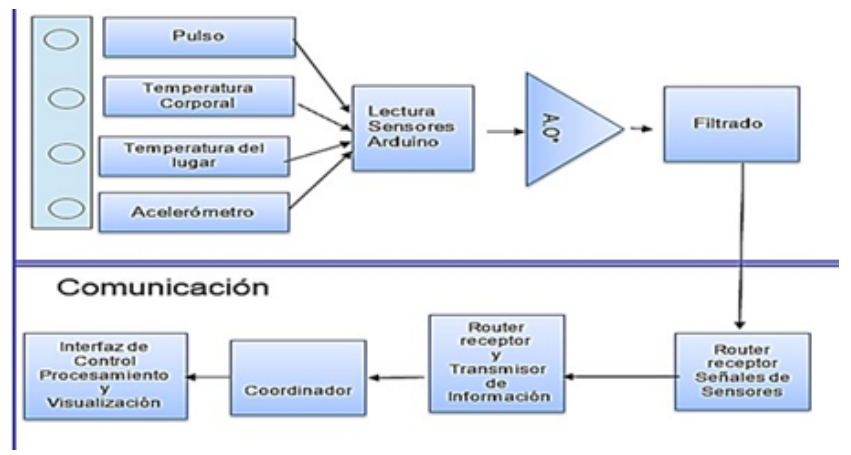

La metodología de este artículo implica cuatro fases:

- Etapa selección de sensores. Tiene en cuenta el rango de sensibilidad y precisión de cada uno y, conforme a ello, se realiza la elección de los diversos sensores como el acelerómetro (MPU 6050), el sensor de temperatura (18B20) y, por último, el sensor de pulso cardiaco (pulse sensor) [14,15].

- Etapa de diseño y desarrollo de fabricación del hardware del primer prototipo experimental no comercial. Se realizan los planos electrónicos y la compatibilidad del sistema de comunicación, el procesamiento de los datos recibidos por cada uno de los sensores mencionados anteriormente.

- Etapa de algoritmo para análisis - a través de filtros digitales - del ruido de la señal y su eliminación, con el fin de visualizarse posteriormente en la plataforma.

- Etapa de ensayos de laboratorio. Se obtienen las pruebas iniciales del prototipo para evaluar el correcto funcionamiento del dispositivo de comunicación y evitar la pérdida de datos del sistema. Atendiendo el decreto 1335 del 15 de julio de 1987 del Ministerio de Minas y Energía, el Ministerio del Trabajo y el Ministerio de Salud y Protección Social, y su actualización - del 21 de septiembre de 2015 - Decreto 1886, "Por el cual se establece el Reglamento de Seguridad en las Labores Mineras Subterráneas", se realiza en la empresa Vertisub - donde existe una cavidad que simula el espacio confinado y una cabina que se encuentra ubicada adyacente al lugar - una prueba de la comunicación a partir de los módems en su configuración WISP (siglas del inglés Wireless Internet Service Provider), trazándose un vínculo de comunicación tipo malla [16-19].

\section{Resultados}

En el caso del acelerómetro, cuando tiene un promedio de datos superior a cero, indica que los movimientos realizados son normales y no ha habido ningún inconveniente ni caída libre, la cual se da cuando los datos son menores a cero [20]. Para el procesamiento de caída libre se tuvo en cuenta que los acelerómetros forman los ángulos $\alpha$ con respecto al eje $x ; \beta$ con respecto al eje $y$; e $\gamma$ con respecto al eje $z$. De acuerdo con esto, las salidas de los acelerómetros se encuentran en (1), (2) y (3).

$$
\begin{aligned}
& \text { Xout }=\text { Xoffset }+\left(\frac{\Delta v}{\Delta g} * 1 g * \sin \alpha\right) \\
& \text { Yout }=\text { Xoffset }+\left(\frac{\Delta v}{\Delta g} * 1 g * \sin \beta\right) \\
& \text { Zout }=\text { Xoffset }+\left(\frac{\Delta v}{\Delta g} * 1 g * \sin \lambda\right)
\end{aligned}
$$

Donde:

Xout, Yout, $Z$ out $=$ salida del acelerómetro en voltios.

Xoffset,Yoffset, Zoffset $=$ valor de offset a $0 \mathrm{~g}$ del acelerómetro.

$\Delta V / \Delta g=$ sensibilidad del acelerómetro.

$1 g=$ gravedad de la tierra [20].

Teniendo en cuenta las fórmulas (1), (2), (3), cuando el objeto cae las aceleraciones en los tres ejes llegarán a valer cero sin importar la orientación del objeto, pues no se detectará ninguna aceleración en ningún eje ya que, como se explicó antes, los acelerómetros caen con la misma aceleración que la ejercida por la fuerza de la gravedad. El algoritmo de detección de caída libre realiza un muestreo de los datos de los acelerómetros y supervisa dichas aceleraciones [20-22].

\subsection{Trazabilidad del personal}

Dentro de los aspectos evaluados en la investigación, se consideró la detección de trazabilidad de las personas que ingresan al espacio confinado, ya que en ocasiones se dificulta saber si el trabajador se encuentra dentro del lugar; por ello, dentro del servidor, se envía un anuncio en caso de no haber emparejamiento, este solo se da con el 
router interno; al salir del módulo ya no detecta conexión y envía un anuncio indicando que el trabajador no se encuentra en el interior del espacio confinado [23, 24].

\subsection{Interface gráfica}

Figura 2: Interface gráfica [1].

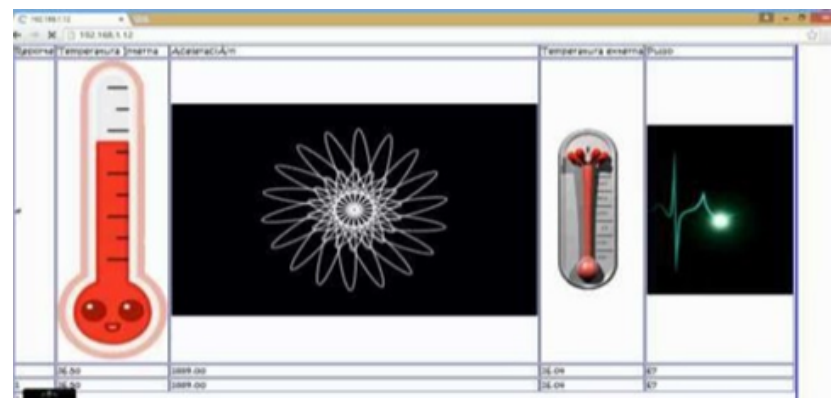

La interface gráfica consta de cuatro imágenes en constante movimiento elaborada en HTML (siglas del inglés HyperText Markup Language; Figura 2), la cual está dividida en cinco columnas, en la primera se visualiza el número de reporte, en la segunda la temperatura corporal, en la tercera el promedio de aceleración, en la cuarta columna la temperatura del espacio confinado y en la quinta el pulso cardiaco en batidos por minuto (BPM).

\subsection{Prototipo}

El prototipo inicial (Figura 3) consta de un artefacto electrónico de $2.5 \mathrm{~cm}$ de ancho y $3.5 \mathrm{~cm}$ de largo, ideal para su fácil portabilidad. En la propuesta inicial se ha optado por ubicar la placa electrónica del módulo en una pequeña caja de material ABS elaborada en fusión 360.

Figura 3: Prototipo inicial terminado.

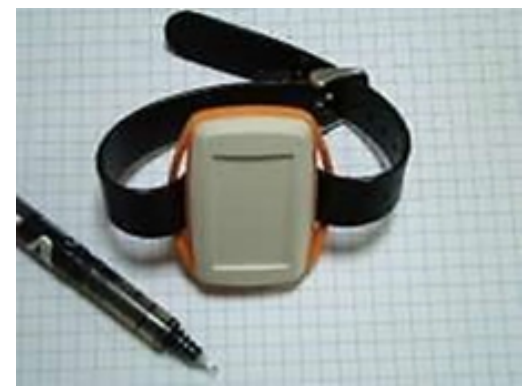

Fuente: elaboración propia.

\section{Conclusiones}

Después de realizar la respectiva revisión bibliográfica y realizar comparaciones desde el criterio de la ingeniería, se identificó que el sistema de comunicación con mejores características para implementar es el Wifi, debido a su alto rango de cobertura y velocidad en el envío de datos, lo que permitió la transmisión de los datos desde el espacio confinado hacia la cabina de monitoreo. Posterior a ello, se logró implementar el dispositivo de adquisición y procesamiento de pulso cardiaco, temperatura corporal, acelerometría y temperatura externa utilizando un conjunto de materiales de bajo costo, con un tamaño adecuado y con un alto rango de precisión lo que le da robustez, viabilidad y funcionalidad al prototipo.

Al contar con los datos procesados y el sistema de comunicación funcional se realizó la respectiva interfaz, que permitió la captura de datos y realizar la respectiva tabulación en cada una de las variables que detecta el sistema; sin embargo, en el caso del sensor de pulso cardiaco se realizó la conversión dada por el algoritmo, donde cada valor de BPM es multiplicado por 125 debido a indicaciones del fabricante.

Uno de los procesos importantes logrados fue determinar la bidireccionalidad del sistema, ya que esto permite que la persona que realiza el monitoreo y el usuario que se encuentra al interior del espacio puedan comunicarse por medio de un estímulo visual que en este caso es un led [25].

Finalmente, después de realizar las respectivas pruebas, se puede concluir que los objetivos de la investigación se cumplieron a cabalidad, desde la identificación del sistema de comunicación, la lectura de los signos vitales y variables externas, culminando con una interfaz que permite comunicación bidireccional.

\section{Recomendaciones}

- Se recomienda seguir con el respectivo mejoramiento y actualización del prototipo, con el fin de que en un futuro próximo logre salir al mercado y tenga una aplicación real en el ámbito de seguridad industrial, el cual actualmente se considera uno de los puntos más importantes respecto al estudio de riesgos laborales [26-28].

- Para llevar a cabo el mejoramiento del dispositivo se considera de importancia realizar pruebas de su calidad y diseñar e implementar algoritmos con menor consumo de memoria para que la transmisión se realice más rápido y se obtengan resultados robustos.

- Se recomienda el mejoramiento de la interfaz gráfica, ya que en la implementación del primer dispositivo se dificulta el manejo de la 
bidireccionalidad desde la misma interfaz, por ello, se requiere abrir otro navegador para proceder a encender el respectivo led.

- El prototipo debe estar conectado a una red que solo sea dedicada para esta aplicación y debe ser superior a $1 \mathrm{~Gb}$ de ancho de banda.

- Colocar una batería que suministre la suficiente corriente para soportar las horas de independencia del dispositivo.

\section{Referencias}

[1] D. G. Cervetto "Monitoreo de gases identifica riesgos en espacios confinados" Revista seguridad minera, $\mathrm{n}^{\circ} 64,2014$.

[2] FITBIT, "Caracteristicas Fitbit". [En línea]. Disponible en: http://www.fitbit.com/es/about

[3] Apple, "Apple Watch" [En línea]. Disponible en: http://www .apple.com/co/watch/

[4] Nike, "Nike Sport Watch". [En línea]. Disponible en: https://secure-nikeplus.nike.com/plus/ products/sport_watch/

[5] UNAD, "Salud Ocupacional". [En línea]. Disponible en: https://saludocupacionalunad.files. wordpress . com/2014/08/102505_modulo_ completo- c.pdf

[6] E. M. Cuenca, "Fundamentos de fisiología", Madrid: Paraninfo, 2006.

[7] A. B. Donnersberger y A. E. Lesak, "Libro de laboratorio de anatomía y fisiología", Barcelona: Paidotribo, 2002.

[8] J. C. Garcia, "Libro de la salud cardiovascular", Madrid: Editorial Nerea, 2009.

[9] S. Walsh, "Diagnostico por el pulso: una guía clínica", Nueva York: Elsevier, 2008.

[10] N. Pizzaro, "Frecuencia cardiaca y pulso". [En línea]. Disponible en: https: //4023molinero.files.wordpress.com/2009/ 09/frecuencia-cardiaca-y-pulso.pdf

[11] V. C. Zegarra, "Alteraciones en el sistema respiratorio". [En línea]. Disponible en: http://www . unjbg.edu.pe/coin2/pdf/01040500303.pdf

[12] M. Á. Arcas, "Fisioterapia respiratoria", Sevilla: MAD Eduforma, 2006.
[13] Y. S. Maldonado, "Sindrome respiratorio". [En línea]. Disponible en: http://es.slideshare.net/ yomimaldonado/toxico-27861501

[14] A. De Paz, "Desarrollo de un sistema de sensores para la detección de sustancias peligrosas", tesis de grado, Tecnología Electrónica y de las Comunicaciones, Universidad Autónoma de Madrid, Madrid, 2015.

[15] J. Maine, "Sensores procesadores de señal". [En línea]. Disponible en: http://arantxa.ii.uam.es/ rdrivera/robotica/h_datos/Sensores_Acond. pdf

[16] H. J. Aguilar, "Sistemas de comunicación digital". [En línea]. Disponible en: http: //agamenon.tsc.uah.es/Asignaturas/ittt/ td/apuntes/Presentacion $\% 20$ tema\%201.pdf

[17] Zigbee Alliance, "ZigBee specification". [En línea]. Disponible en: www.zigee.org

[18] IEEE, “ Wireless Medium Access Control (MAC) and Physical Layer (PHY) Specifications for Low-Rate". [En linea]. Disponible en: https:// ieeexplore.ieee.org/document/4152704/

[19] D. E. Cuji y D. X. Pazmiño, "Diseño e implementación de una red WSN (Wireless Sensor Network) basado en los protocolos Zigbee, Wifi y Zigbee Mesh, para el monitoreo de variables climáticas en el invernadero ubicado en el barrio Rumipamba del Navas, cantón Salcedo, provincia de Cotopaxi", tesis de grado, Eléctrica e Instrumentación, Universidad de las Fuerzas Armadas, Latacunga, 2015.

[20] R. Gonález. "Tecnologías Inalambricas", Seguridad y Medio Ambiente, $\mathrm{n}^{\circ}$. 125, 2012

[21] Hetpro, "Acelerometro", [En línea]. Disponible en: http://hetpro-store.com/TUTORIALES/ acelerometro-mpu-6050-6dof /

[22] V. García. "Acelerómetro procesamiento". [En línea]. Disponible en: http://hispavila.com/blog/ el-sensor-mpu-6050/

[23] Cooperativas de Galicia, "Espacios confinados". [En línea]. Disponible en: http://www. cooperativasdegalicia.com/imagenes/ programas/200502181224540.MANUAL_DE_ ESPACIOS_CONFINADOS.pdf

[24] J. G. Escribano, A. García y M. Fuente," Monitorización de la Condición Física de Personas en Espacios Confinados Mediante Etiquetas RFID 
con Sensores y Redes Inalámbricas Eficientes", Revista Iberoamericana de Automática e Informática Industrial RIAI, pp. 371-384, 2011.

[25] Esport. "Bidireccionalidad", [En línea]. Disponible en: http://www.eutanasia.ws/hemeroteca/ escuchaactiva.pdf

[26] M. Fuentes, F. Zuñiga, F. Rodríguez y C. Montero, "Actividad fisica laboral y composición corporal en mujeres adultas", Nutrición Hospitalaria, vol. 28, $\mathrm{n}^{\circ}$. 4, 2013.

[27] N. Garatachea, G. Torres y J. G. Gallego, "Physical activity and energy expenditure measurements using Accelerometers in older adults", Nutrición Hospitalaria, vol. 25, $\mathrm{n}^{\circ}$. 2, 2010.

[28] J. C. Rubio y M. C. Rubio, "Manual de coordinación de seguridad y salud en las obras de construcción", Madrid: Ediciones Diaz de Santos, 2005, pp. 483-484. 